\title{
New Characteristics of Active Life Expectancy of the Elderly in China
}

\author{
Ping Gao, Han-Dong $\mathrm{Li}^{*}$ \\ Department of Management Science and Engineering, School of Government, Beijing Normal University, \\ Beijing, China \\ Email: gaoping4069111@126.com, "Ihd@bnu.edu.cn
}

Received 18 December 2015; accepted 25 January 2016; published 28 January 2016

Copyright () 2016 by authors and Scientific Research Publishing Inc.

This work is licensed under the Creative Commons Attribution International License (CC BY).

http://creativecommons.org/licenses/by/4.0/

(c) (1) Open Access

\section{Abstract}

Life expectancy of the elderly is a significant problem in China, and it changes not only the health care, but also the pension. This study used tracking data from the Chinese Urban and Rural Elderly Population Survey to calculate the age-specific Active Life Expectancy (ALE) of the Chinese elderly population aged 60 years and over. For analysis, this population was divided into different subpopulations according to gender, census register and region. The main conclusions of our study are as follows: 1) The quality of life for elderly males may be greater than that for elderly females; 2) There were significant differences in Active Life Expectancy (ALE) and Inactive Life Expectancy (ILE) between urban and rural elderly; 3) The differences in ALE between the eastern, central and western regions of China were not significant; and 4) The increased remaining life expectancy of the elderly was mainly attributed to the extended ALE in the lower age group and the expanded ILE in the higher age group. This study expands the knowledge of Chinese elderly's life expectancy in different health status.

\section{Keywords}

Aging, Remaining Life Expectancy, Active Life Expectancy (ALE), Inactive Life Expectancy (ILE), Multistate Life Table

\section{Introduction}

Aging is one of the greatest challenges in the 21st century in China. Data from the 2010 Sixth National Population Census showed that the Chinese population aged 60 years and above reached 177.6 million, which

\footnotetext{
*Corresponding author.
} 
represents $13.26 \%$ of the total population, and that the Chinese population aged 65 years and above reached 118.8 million, or $8.87 \%$ of the total population. These proportions are expected to increase rapidly over the next 40 years.

Thus, the following question has arisen in regards to quality of life: Are these additional years of life spent in good health or in a prolonged state of illness and dependency? In an aging society, remaining life expectancy, self-care status and changing trends constitute the major concerns, as it will be important to form expectations not only about how long people are expected to live but also about how healthy they will be in the future. The health conditions of the elderly not only are the basic indicators of their quality of life but also represent important indicators of their social and economic development levels, and of the pension and health care expenditures of their countries and families [1]-[4].

Another important question in aging populations is whether increases in life expectancy will be accompanied by more or fewer life years spent in poor health and/or with disability [4]. Disability, especially as indicated by limitations in carrying out activities of daily living (ADLs), such as bathing, dressing and eating [5], is the most commonly used population-level summary measure of health in late life. Various frameworks for understanding disability have been proposed [6] [7], with one common element being the conceptualization of disability as a gap between individual capacities and the demands of performing a task within a particular environment. A related element in some of these frameworks is the progression from pathology and impairment at the cellular and organ levels (e.g., arthritis and difficulty of bending a knee) to limitation in functioning at the individual level (e.g., crouching) and disability in carrying out a task (e.g., bathing) in a particular environmental context (e.g., a walk-in shower versus a bathtub) [6] [8]. ADL disability is viewed as the culmination of the disablement process and typically represents more severe limitations than simple physical functioning or even performing instrumental ADL, which includes routine household tasks.

Active life expectancy (ALE) (or disability-free life expectancy) typically combines mortality and morbidity information to represent overall population health in a single indicator. Specifically, ALE measures the number of remaining life years that a person at a certain age is expected to live without poor health and is increasingly used to complement the conventional measure of life expectancy [9]. Because health expectancy was developed to reflect that not all years of a person's life are lived in perfect health, and estimates of health expectancies have been very attractive and the widely used tools for monitoring trends in population health [10].

Studies on health among the Chinese elderly have focused on indicators and methods that measure their health. For instance, Gu and Zeng provided a brief introduction of the methods for calculating healthy life expectancy and analyzing the advantages and disadvantages of these methods [11]. Zeng Yi et al. offered a new method for correcting the deviation in elderly life expectancy estimated by complete daily life self-care (called healthy life expectancy) and disability self-care (called unhealthy life expectancy) [12] [13]. These results showed that healthy and unhealthy life expectancies were closely related to life self-care ability at the beginning age. Other studies have focused on subpopulation differences in healthy life expectancy. For example, Gao found that the proportion of elderly females with diseases at earlier ages was greater than that of elderly males [14], and Zeng Yi et al. revealed that the probability that elderly individuals will experience mild pain until death is greater for males than for females, whereas the probability that elderly individuals will experience long-term severe pain until death is lower for males than for females [15]. Du and Li showed that from 1994 to 2004, the Chinese elderly's life expectancy and life self-care expectancy both increased, while the proportion of life self-care expectancy to life expectancy decreased [1]. Generally, the degree of health improvement is less than the degree of increased longevity. Gao used Chinese Elderly Population Health Survey data from 2002 to 2005 to investigate differences between rural and urban elderly populations in China, and their results showed that the ratio of healthy life expectancy in every age group in the rural elderly population was higher than that in the urban elderly population [14].

We used 2006 and 2010 tracking data from the Chinese Urban and Rural Elderly People Survey, which was administered by the China Research Center on Aging that opened in 2012, and IMaCh, a method that uses multistate life tables [16], to calculate the age-specific remaining life expectancy and healthy status of Chinese elderly.

The remainder of this paper is structured as follows. Section 2 introduces the multistate life table and the IMaCh method; section 3 explains the data sources and data processing method; section 4 provides an empirical study of the 2006 and 2010 tracking data; and section 5 presents our conclusions. 


\section{Methods}

\subsection{Multistate Life Table}

There are multiple ways to calculate healthy life expectancy, such as the Sullivan method, the double-decrement life table model, the increment-decrement life table method, the microscopic simulation method and the membership grade model method; below, we review the advantages and disadvantages of these methods [3] [11] [15].

Multistate life table methods are used in demography to estimate the length of remaining life that individuals can expect to live in different states, such as healthy versus unhealthy and married versus unmarried. One of the most common applications of this method has been the estimation of healthy life expectancy, defined as the length or proportion of remaining life spent free from disability, chronic disease, or other health problems. One specific focus of multistate methods has been to estimate ALE, which is the length of life spent free from physical limitations. To date, the method most often used to estimate ALE has been Sullivan's method [17], which is not a true multistate method but which provides good estimates of ALE with less stringent data requirements than true multistate approaches [18] [19].

Our main aim was to use longitudinal tracking data from 2006 and 2010 on Chinese urban and rural elderly individuals to study life expectancy along certain health dimensions. To compensate for the method's weakness of only examining two transfers at the survey point, which means that the method cannot reveal more than one transfer between any two survey points, we used interpolated Markov chains (IMaCh) (Laditka and Wolf, 1998) to consider a variety of healthy state transitions between the two survey points.

\subsection{IMaCh}

IMaCh was proposed by Laditka and Wolf (1998) to calculate the transition probabilities of multistate life tables, and this approach can overcome three problems encountered in survey data. First, the tracking survey interval is commonly 2 years or longer, and most studies assume that respondents experience at most one state transition during the survey interval; obviously, this assumption is not very credible. Second, the survey intervals between respondents are different and can be as long as one year within the same survey. Third, because of cost, the survey time is generally short, and only limited data can be obtained to calculate life expectancy.

IMaCh first uses the maximum likelihood method to obtain a matrix of transition probabilities and then uses the original survey data to simulate the life cycle data and thereby obtain more life table parameter data. This approach solves the problem of insufficient data across the life cycle, so we use this software to obtain the life expectancy, ALE and ILE of different elderly people.

This software is described at the following website: $\mathrm{http} / / /$ euroreves.ined.fr/imach/doc/imach.htm.

\section{Data}

\subsection{Data Description}

The data for this study were obtained from the 2006 and 2010 Chinese Urban and Rural Elderly Population Surveys, conducted by the China Research Center on Aging of the National Committee on Aging. These data cover the following 20 provinces, municipalities and autonomous regions in China: North China-Beijing, Hebei, and Shanxi; Northeast China-Liaoning and Heilongjiang; East China-Shanghai, Jiangsu, Zhejiang, Anhui, Fujian, and Shandong; Mid-South China-Henan, Hubei, Hunan, Guangdong, and Guangxi; Southwest China-Sichuan and Yunnan; and Northwest China-Shanxi and Xinjiang. The data sampling method was the same as for the Fifth Population Census; based on the distribution of the population 60 years and older, a quota from each of the six regions (province, municipalities and autonomous regions) could be determined. Then, stratified sampling was used to confirm that the survey results represented the total elderly population in China. The main subject of investigation in 2010 was the same elderly population investigated in 2006; these two surveys obtained samples of 19,947 person-times and 19,986 person-times, respectively. The investigation subjects included elderly individuals ranging from 60 to 102 years in age. Between the two surveys, effective tracking data on the elderly were available for $15,036(12,530+2506)$ person-times, of which 12,530 were alive in both surveys and 2506 had died in the second survey. Table 1 shows the statistics for the original data. 
Table 1. Tracking data statistics.

\begin{tabular}{|c|c|c|c|c|c|}
\hline Status & $\begin{array}{c}\text { Original } \\
\text { tracking } \\
\text { number }\end{array}$ & Unqualified data description & $\begin{array}{c}\text { Effective } \\
\text { tracking } \\
\text { number }\end{array}$ & Further screening principle & $\begin{array}{c}\text { Qualified } \\
\text { screening } \\
\text { number }\end{array}$ \\
\hline Survivors & 12,699 & $\begin{array}{l}\text { a. Repeat case: } 25 \\
\text { b. Tracking individuals have no } \\
\text { data: } 40 \\
\text { c. Survivors have death registration: } \\
104\end{array}$ & 12,530 & $\begin{array}{l}\text { a. Default-free } \\
\text { b. Gender, province, census } \\
\text { register consistent } \\
\text { c. Age gap is } 3 \text { to } 5 \\
\text { d. Education gap is positive }\end{array}$ & 8703 \\
\hline Death & 2539 & a. Repeat case: 13 & 2506 & a. Default-free & 1742 \\
\hline
\end{tabular}

\subsection{Data Processing}

To apply the multistate life table method more appropriately, we processed the essential data from both surveys to accurately reflect the health conditions of the elderly. The required data samples also needed to track longitudinal survey data; thus, correctly connecting the data from the two surveys was the key aim in data processing.

1) All of the survey indicators had to be default-free.

2) The connection between the data from the two surveys was based on the 2010 questionnaire. We discarded samples with inconsistent gender, province, and census register results (e.g., an individual who transferred from an agricultural to a nonagricultural area was considered to live in a nonagricultural census register) and also eliminated data with a negative value for the two-year gap in education level.

3) Based on native Chinese cultural use of the lunar calendar, the age gap was limited to 3 to 5 years, and we discarded data samples beyond that gap.

4) To facilitate the distinction between urban and rural, we used the agricultural and nonagricultural census registers.

5) We enrolled 2,006 participants who died between 2006 and 2010 and included these data in the overall sample data, excluding the samples for which death was recorded but not a date of death.

6) As we determined the health status of each sample, if there was a default value for the six self-care health indicators, the sample was eliminated.

7) Because only the year of birth was recorded, to obtain accurate dates of birth, we used random numbers between 1 and 12 as the sample's birth month and combined that number with the year of birth as the essential data.

8) For death records that only included the year of death, we used a random number between 1 and 12 as each sample's death month and combined that with the year of death as the essential data.

9) To ensure that the proportion of survival and death screening samples was consistent with the proportions in the original survival and death samples $(12,530: 2506=5: 1)$, when the number of screening survival samples was 8703 , we randomly chose $8703 / 5=1741$ samples from the 2506 death samples as the final death sample data.

10) The 20 provinces, municipalities and autonomous regions were divided into the following groups: eastern—Beijing, Shanghai, Hebei, Liaoning, Jiangsu, Zhejiang, Fujian, Shandong, and Guangdong; central-Heilongjiang, Anhui, Henan, Shanxi, Hubei, and Hunan; and western-Sichuan, Yunnan, Shaanxi, Xinxiang, and Guangxi.

Using SPSS software to realize the above-described processing methods, the final available number of samples was 10,444, including 5512 records for male elderly and 4932 records for female elderly; 4751 records from nonagricultural census registers and 5693 records from agricultural census registers; and 4649 records from the eastern region, 3106 records from the central region, and 2589 records from the western region.

\subsection{Definition of Health Indicators}

ADLs are used to measure the elderly's ability to take care of themselves in certain aspects of daily life, including eating, dressing, bathing, using the toilet, indoor activities, and continence [20]. We adopted six indicators to evaluate the samples' health conditions, with three rating options for each indicator: effortlessly, with some trouble and cannot do it. A person was considered completely able to manage self-care, or healthy, when he or she had recorded difficulty with at most one indicator but the other indicators were rated effortless. Otherwise, 
the subject was considered not to have full capacity for independent living, or unhealthy.

\section{Data Analysis Results}

The data we processed could be divided into different subsamples according to gender, census register and region, which allowed us to calculate the Chinese elderly's state transition probabilities. Then, using the multistate life table method and IMaCh, we obtained the remaining life expectancy, ALE, ILE and healthy life expectancy proportions among the different subpopulations as well as any age-related trends over time in these four indicators.

\subsection{Age-Specific State Transition Probabilities of the Elderly}

Table 2 presents the state transition probabilities of the Chinese elderly population aged 60 to 100 years, with age intervals of 5 years. P11 represents the probability of maintaining the active state (active state to active state); P12 represents the probability of transferring from the active state to the inactive state; and P13 represents the probability of transferring from the active state to the death state. An active or healthy person at any moment could be in only one of the three states: maintaining the active state, transferring to the inactive state, or transferring to death. Therefore, $\mathrm{P} 11+\mathrm{P} 12+\mathrm{P} 13=1$. $\mathrm{P} 21$ represents the probability of transiting from the inactive to the active state; P22 represents the probability of maintaining the inactive state; and P23 represents the probability of transiting from the inactive to the death state, with P21 + P22 + P23 = 1. Here, the unit of all transfer intervals between states is one year; in other words, the transition probability is the probability that one elderly person will transit from one state to another within one year.

Combining Table 2, we observed the following similarities in the state transition probabilities of the different genders. 1) P11 decreased as age increased for both male and female elderly. That is, among those whose initial

Table 2. Tracking data statistics.

\begin{tabular}{|c|c|c|c|c|c|c|}
\hline Age & P11 & $\mathrm{P} 12$ & P13 & P21 & P22 & P23 \\
\hline \multicolumn{7}{|c|}{ Male elderly } \\
\hline 60 & 0.9840 & 0.0071 & 0.0088 & 0.1674 & 0.7723 & 0.0603 \\
\hline 65 & 0.9730 & 0.0131 & 0.0139 & 0.1394 & 0.7806 & 0.0801 \\
\hline 70 & 0.9545 & 0.0239 & 0.0216 & 0.1147 & 0.7801 & 0.1052 \\
\hline 75 & 0.9238 & 0.0430 & 0.0333 & 0.0933 & 0.7703 & 0.1365 \\
\hline 80 & 0.8744 & 0.0756 & 0.0500 & 0.0748 & 0.7504 & 0.1747 \\
\hline 85 & 0.7990 & 0.1283 & 0.0727 & 0.0591 & 0.7204 & 0.2205 \\
\hline 90 & 0.6930 & 0.2067 & 0.1003 & 0.0460 & 0.6804 & 0.2737 \\
\hline 95 & 0.5605 & 0.3106 & 0.1289 & 0.0351 & 0.6312 & 0.3337 \\
\hline 100 & 0.4175 & 0.4297 & 0.1527 & 0.0263 & 0.5745 & 0.3992 \\
\hline \multicolumn{7}{|c|}{ Female elderly } \\
\hline 60 & 0.9847 & 0.0098 & 0.0055 & 0.2148 & 0.7547 & 0.0305 \\
\hline 65 & 0.9740 & 0.0177 & 0.0083 & 0.1710 & 0.7856 & 0.0433 \\
\hline 70 & 0.9558 & 0.0318 & 0.0123 & 0.1341 & 0.8052 & 0.0607 \\
\hline 75 & 0.9254 & 0.0565 & 0.0181 & 0.1035 & 0.8127 & 0.0838 \\
\hline 80 & 0.8762 & 0.0979 & 0.0259 & 0.0787 & 0.8075 & 0.1138 \\
\hline 85 & 0.8003 & 0.1638 & 0.0359 & 0.0588 & 0.7892 & 0.1520 \\
\hline 90 & 0.6931 & 0.2598 & 0.0471 & 0.0432 & 0.7574 & 0.1994 \\
\hline 95 & 0.5589 & 0.3837 & 0.0575 & 0.0311 & 0.7125 & 0.2564 \\
\hline 100 & 0.4144 & 0.5211 & 0.0645 & 0.0219 & 0.6556 & 0.3225 \\
\hline
\end{tabular}


state was active, and with age increasing, the probability that they would maintain the active state decreased. In addition, P12 and P13 increased as age increased. That is, among the elderly whose initial state was active, with increased age, the probabilities that they would transit from the active to the inactive state and to the death state both increased. Meanwhile, line P12 was always above line P13 with increased age, and the gap between line P12 and P13 increased gradually. That is, among those whose initial state was active, the probability that they would transit from the active to the inactive state was greater than that of their transition from the active to the death state, and the increasing amplitude of the probability of transiting from the active to inactive state was greater than that of transiting from the active state to death. 2) P21 decreased as age increased for male and female elderly. In addition, P23 increased as age increased. That is, among the elderly whose initial state was inactive, with increased age, their probability of transiting to the active state decreased, and the probability of their transition to the death state increased. For males below 70 years and females below 77 years, the probability of their transition from the inactive to active state was greater than that of their transition from the inactive state to death. For the older elderly, the probabilities were the opposite. 3) Line P22 was monotonous with increased age. There was first a small amplitude increase and then a gradual decrease.

Although the overall trends for both genders were similar, the following differences were also observed. 1) Lines P13 and P23 for males were always above those for females, indicating that for both the active and the inactive elderly, males were more likely to transit to the death state than females. 2) Line P12 for males was always below that for females, which reflects that among the active elderly, females were more likely than males to transition to the inactive state. 3) Line P22 for both males and females was monotonous, although there were obvious differences. Line P22 for males remained approximately unchanged and then decreased gradually with increasing age, while there was a clear increase in line P22 for females that subsequently decreased. The line P22 turning point was age 70 for males and age 77 for females. Line P22 for females was always above that for males, indicating that among the inactive elderly, females were more likely to maintain the inactive state than males.

\subsection{Age-Specific Life Expectancy by Gender}

Table 3 presents the remaining life expectancy, ALE, ILE and healthy life expectancy proportions among the Chinese elderly aged 60 to 100 years, with age intervals of 5 years. Here age-specific life expectancy represents the sum of ALE and ILE. To facilitate this comparison, we used the ALE proportion as an indicator because it reflects the contribution of ALE to remaining life expectancy. From Table 3, we can see that for males aged 60 years, life expectancy was 20.20 years, ALE was 17.53 years, and ILE was 2.67 years; therefore, the ALE proportion was $17.53 / 20.20=86.77 \%$. For females aged 60 years, life expectancy was 23.65 years, ALE was 18.75 years, ILE was 4.90 years, and the ALE proportion was $78.28 \%$.

Figure 1(a) shows the life expectancy, ALE and ILE of the elderly according to age and gender, and Figure

Table 3. Age-specific healthy and unhealthy life expectancy by gender.

\begin{tabular}{|c|c|c|c|c|c|c|c|c|}
\hline \multirow{3}{*}{ Age } & \multicolumn{4}{|c|}{ Male } & \multicolumn{4}{|c|}{ Female } \\
\hline & \multicolumn{3}{|c|}{ Life expectancy (year) } & \multirow{2}{*}{ ALE proportion $(\%)$} & \multicolumn{3}{|c|}{ Life expectancy (year) } & \multirow{2}{*}{$\begin{array}{c}\text { ALE proportion } \\
(\%)\end{array}$} \\
\hline & Active & Inactive & Total & & Active & Inactive & Total & \\
\hline 60 & 17.53 & 2.67 & 20.20 & 86.77 & 18.75 & 4.90 & 23.65 & 79.28 \\
\hline 65 & 13.61 & 2.70 & 16.31 & 83.45 & 14.55 & 4.91 & 19.46 & 74.78 \\
\hline 70 & 10.07 & 2.72 & 12.79 & 78.77 & 10.67 & 4.87 & 15.54 & 68.67 \\
\hline 75 & 7.01 & 2.71 & 9.72 & 72.12 & 7.26 & 4.74 & 12.00 & 60.49 \\
\hline 80 & 4.51 & 2.66 & 7.17 & 62.87 & 4.47 & 4.48 & 8.95 & 49.96 \\
\hline 85 & 2.63 & 2.56 & 5.19 & 50.72 & 2.43 & 4.06 & 6.49 & 37.48 \\
\hline 90 & 1.36 & 2.38 & 3.74 & 36.42 & 1.15 & 3.51 & 4.66 & 24.67 \\
\hline 95 & 0.62 & 2.12 & 2.74 & 22.59 & 0.48 & 2.92 & 3.40 & 14.18 \\
\hline 100 & 0.27 & 1.83 & 2.10 & 12.64 & 0.20 & 2.36 & 2.56 & 7.67 \\
\hline
\end{tabular}




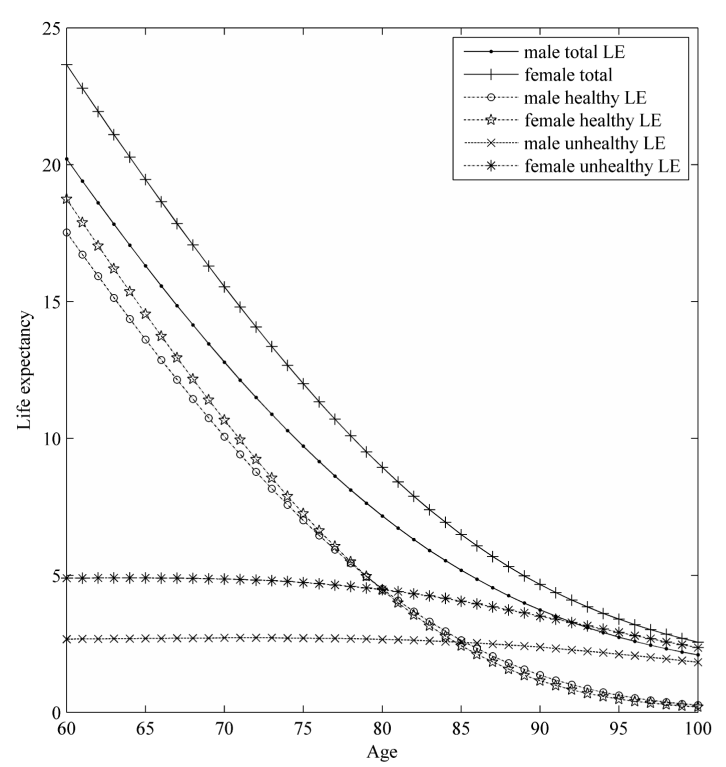

(a)

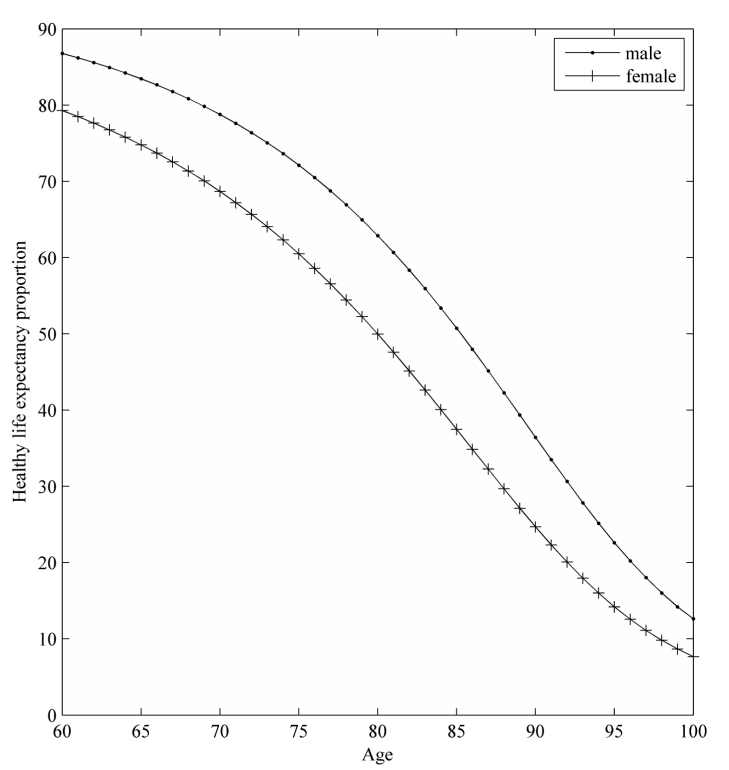

(b)

Figure 1. Life expectancy, ALE and ILE (left) and ALE proportions (right) of the different genders.

1(b) shows the different ALE proportions, also according to age and gender.

From Figure 1(a), we can clearly see that life expectancy and ALE decreased rapidly when age increased but that ILE was stable; only in the higher ages ( 85 and over for males and 75 and over for females) did ILE gradually decrease. This finding indicates that the changes in the male and female elderly's life expectancies were caused by changing ALE, whereas ILE remained approximately unchanged. At later ages, ALE tended to be zero with increasing age, such that life expectancy was approximately equivalent to ILE. This result helps elucidate life expectancy structure.

Comparing the differences between male and female elderly, we can see that within the same age groups, life expectancy and ALE for elderly females were greater than those for elderly males and that the gap decreased as age increased; among the 100-year-old age group, this gap was less than six months, and ALE was approximately zero. Furthermore, we found that the females' life expectancy and ALE were both greater than those for males but that the ILE for female elderly was clearly greater than that for male elderly. For example, among those aged 70 years, life expectancy for males and females was 12.79 and 15.54 years, respectively, with a gap of 2.75 years. In addition, the ALE for males and females was 10.07 and 10.67 years, respectively, with a gap of 0.6 years, and the ILE was 2.72 and 4.87 years, respectively, with a gap of 2.15 years. The gap in ILE accounted for $78 \%$ of the gap in life expectancy. At aged 70 years, the ALE proportions for males and females were $78.77 \%$ and $68.67 \%$, respectively.

The differences in ALE proportions between males and females can be seen in Figure 1(b). Although the two lines show the same tendency, the line for males is clearly above that for females, indicating that quality of life for elderly males was significantly higher than that for elderly females, although the females' life expectancy and ALE were clearly greater than those for males.

\subsection{Age-Specific Life Expectancy by Census Register}

Table 4 and Table 5 provide the life expectancy, ALE, ILE and ALE proportions according to census register and gender for the Chinese elderly aged 60 to 100 years, with an age interval of 5 years.

China utilizes a dual-structure (urban vs. rural) census register system in which residents are divided into agricultural (mainly living in the country) or nonagricultural areas (mainly living in cities and towns). Because urban and rural areas have different economic development levels, resources and environmental conditions, we sought to identify any differences between the residents of these two areas.

As shown in Table 4 and Table 5, there were significant differences between the urban and rural elderly. For example, for 60 -year-olds, the life expectancies of urban vs. rural elderly males were 23.16 and 18.54 years, 
Table 4. Age-specific healthy and unhealthy life expectancy by census register (urban).

\begin{tabular}{|c|c|c|c|c|c|c|c|c|}
\hline \multirow{3}{*}{ Age } & \multicolumn{4}{|c|}{ Male } & \multicolumn{4}{|c|}{ Female } \\
\hline & \multicolumn{3}{|c|}{ Life expectancy (year) } & \multirow{2}{*}{ ALE proportion (\%) } & \multicolumn{3}{|c|}{ Life expectancy (year) } & \multirow{2}{*}{$\begin{array}{c}\text { ALE proportion } \\
(\%)\end{array}$} \\
\hline & Active & Inactive & Total & & Active & Inactive & Total & \\
\hline 60 & 20.05 & 3.11 & 23.16 & 86.57 & 20.73 & 4.48 & 25.21 & 82.21 \\
\hline 65 & 15.89 & 3.11 & 19.00 & 83.62 & 16.25 & 4.49 & 20.74 & 78.37 \\
\hline 70 & 12.08 & 3.14 & 15.22 & 79.37 & 12.03 & 4.45 & 16.48 & 72.99 \\
\hline 75 & 8.72 & 3.19 & 11.91 & 73.23 & 8.22 & 4.34 & 12.56 & 65.41 \\
\hline 80 & 5.90 & 3.25 & 9.15 & 64.48 & 5.03 & 4.12 & 9.15 & 54.99 \\
\hline 85 & 3.69 & 3.30 & 6.99 & 52.83 & 2.66 & 3.72 & 6.38 & 41.66 \\
\hline 90 & 2.15 & 3.29 & 5.44 & 39.51 & 1.19 & 3.19 & 4.38 & 27.23 \\
\hline 95 & 1.24 & 3.20 & 4.44 & 28.02 & 0.48 & 2.59 & 3.07 & 15.53 \\
\hline 100 & 0.80 & 3.01 & 3.81 & 21.06 & 0.20 & 2.06 & 2.26 & 9.00 \\
\hline
\end{tabular}

Table 5. Age-specific healthy and unhealthy life expectancy by census register (rural).

\begin{tabular}{|c|c|c|c|c|c|c|c|c|}
\hline \multirow{3}{*}{ Age } & \multicolumn{4}{|c|}{ Male } & \multicolumn{4}{|c|}{ Female } \\
\hline & \multicolumn{3}{|c|}{ Life expectancy (year) } & \multirow{2}{*}{ ALE proportion (\%) } & \multicolumn{3}{|c|}{ Life expectancy (year) } & \multirow{2}{*}{$\begin{array}{l}\text { ALE proportion } \\
(\%)\end{array}$} \\
\hline & Active & Inactive & Total & & Active & Inactive & Total & \\
\hline 60 & 15.93 & 2.61 & 18.54 & 85.92 & 17.03 & 5.25 & 22.28 & 76.44 \\
\hline 65 & 12.15 & 2.65 & 14.80 & 82.12 & 13.13 & 5.27 & 18.40 & 71.35 \\
\hline 70 & 8.77 & 2.66 & 11.43 & 76.73 & 9.58 & 5.23 & 14.81 & 64.68 \\
\hline 75 & 5.90 & 2.63 & 8.53 & 69.21 & 6.51 & 5.09 & 11.60 & 56.14 \\
\hline 80 & 3.64 & 2.52 & 6.16 & 59.09 & 4.04 & 4.80 & 8.84 & 45.75 \\
\hline 85 & 2.02 & 2.32 & 4.34 & 46.47 & 2.26 & 4.35 & 6.61 & 34.17 \\
\hline 90 & 0.99 & 2.05 & 3.04 & 32.53 & 1.12 & 3.78 & 4.90 & 22.83 \\
\hline 95 & 0.43 & 1.74 & 2.17 & 19.73 & 0.50 & 3.18 & 3.68 & 13.58 \\
\hline 100 & 0.17 & 1.44 & 1.61 & 10.48 & 0.21 & 2.61 & 2.82 & 7.49 \\
\hline
\end{tabular}

respectively, with a gap of 4.62 years; their ALEs were 23.16 and 18.54 years, respectively, with a gap of 4.12 years; their ILEs were 3.11 and 2.61 years, respectively, with a gap of 0.5 years; and their ALE proportions were $86.57 \%$ and $76.44 \%$, respectively. For female elderly, urban vs. rural life expectancies were 25.21 and 22.28 years, respectively, with a gap of 2.93 years; the ALEs were 20.73 and 17.03 years, respectively, with a gap of 3.7 years; the ILEs were 4.48 and 5.25 years, respectively, with a gap of -0.77 years; and their ALE proportions were $82.21 \%$ and $76.44 \%$, respectively.

Figure 2(a) presents the different life expectancies according to census register and gender; Figure 2(b) shows the ALEs of the different groups; Figure 2(c) shows their different ILEs; and Figure 2(d) presents the different groups' ALE proportions.

Furthermore, our results led us to the following conclusions. 1) These four indicators among the urban vs. rural elderly showed the same tendencies as those for the elderly nationwide, but there were also differences. For instance, in the lower age group, urban males had a lower life expectancy and ALE than did urban females, whereas in the higher age group, these two indicators were both greater for urban males than for urban females. The life expectancy turning point was 80 years of age, and that for ALE was 68 years of age. 2) There were significant differences between the urban and rural elderly on all four indicators, with the values for the urban elderly greater than those for their rural counterparts. Urban and rural areas are subject to different economic development levels, job conditions and lifestyles. Thus, additional analysis will help to further elucidate the factors that influence the life expectancies and health conditions of the Chinese elderly. 3) There was a meaningful 


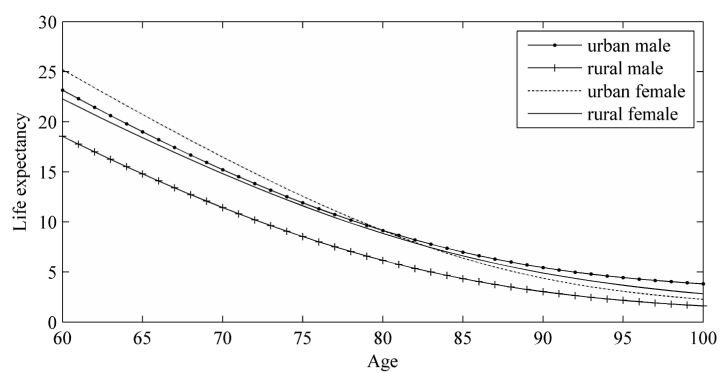

(a)

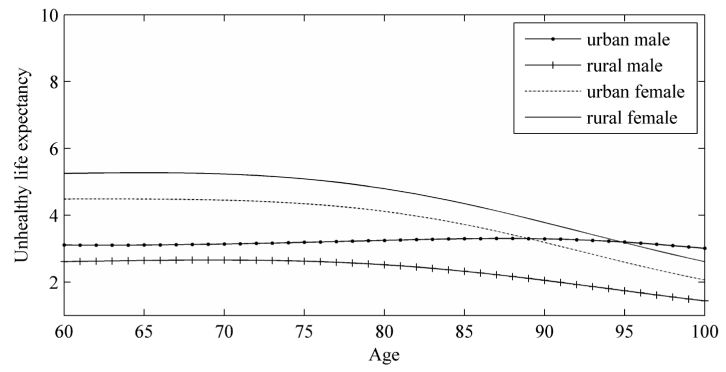

(c)

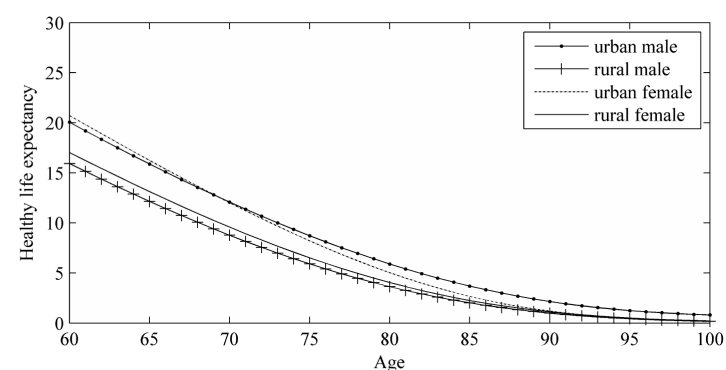

(b)

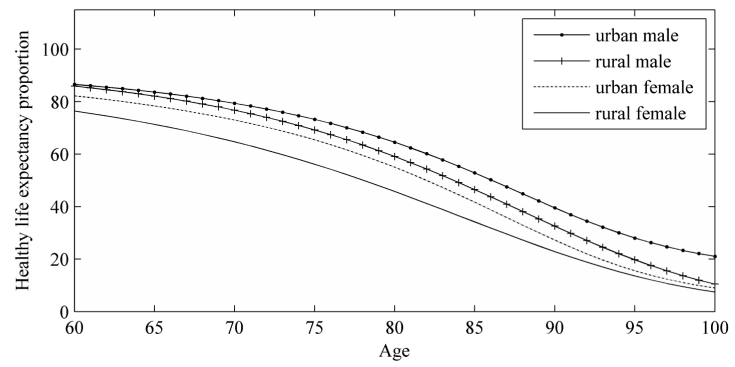

(d)

Figure 2. Life expectancy (a), ALE (b), ILE (c) and ALE proportion (d) by census register.

finding regarding the ILEs of the urban and rural elderly males. In the lower age group, the ILEs for the urban and rural elderly males remained approximately unchanged, and the gap between them was fixed. In the higher age group, the ILEs for urban males remained mostly stable with a small increase, but the ILEs for rural elderly males showed a significant decrease. Additionally, in the higher age group, the ILEs of both urban and rural elderly males were approximately zero, and thus, the difference in life expectancy was the difference in ILE. This finding could be mainly explained by the differences in lifestyle and medical treatment options between urban and rural areas. Therefore, in-depth study of the differences between these two groups is necessary.

In conclusion, our analysis indicates that the Chinese elderly's life expectancy and health conditions reflect obvious differences between urban and rural areas. Moreover, by comparing urban and rural elderly, we can conclude that increased life expectancy depends mainly on increased ALE in the lower age group but mainly on increased ILE in the higher age group.

\subsection{Age-Specific Life Expectancy by Region}

The significant differences between the life expectancy and health conditions of urban and rural elderly led us to examine the differences between the country's regions. Therefore, based on the current widely used division standard (the eastern, central and western regions), which reflects the regional economic differences in China, the country's 20 provinces, municipalities and autonomous regions were divided into three parts: eastern-Beijing, Shanghai, Hebei, Liaoning, Jiangsu, Zhejiang, Fujian, Shandong, and Guangdong; central-Heilongjiang, Anhui, Henan, Shanxi, Hubei, and Hunan; and western-Sichuan, Yunnan, Shanxi, Xinxiang, and Guangxi. This geographic division mainly accounts for differences in regional economic and social development; for instance, the eastern region includes China's economically developed coastal provinces of Beijing, Shanghai and Guangdong. Additionally, the economic development level of the central region is lower than that of the east, and the western region is generally underdeveloped. Comparison of these three regions was performed to reveal the relationship between economic development level and life expectancy and health conditions among the elderly.

Tables 6-8 present the life expectancy, ALE, ILE and ALE proportions of China's elderly by region and gender from age 60 to age 100, with age intervals of 5 years. Partial data in the tables show that there were significant differences between regions; in particular, the eastern region's indicators were significantly higher than those for the central region, and the central region's indicators were significantly higher than those for the western region. For instance, for male elderly at 60 years of age in the eastern, central and western regions, the life 
Table 6. Age-specific healthy and unhealthy life expectancies of the elderly in the eastern region.

\begin{tabular}{|c|c|c|c|c|c|c|c|c|}
\hline \multirow{3}{*}{ Age } & \multicolumn{4}{|c|}{ Male } & \multicolumn{4}{|c|}{ Female } \\
\hline & \multicolumn{3}{|c|}{ Life expectancy (year) } & \multirow{2}{*}{ ALE proportion (\%) } & \multicolumn{3}{|c|}{ Life expectancy (year) } & \multirow{2}{*}{$\begin{array}{l}\text { ALE proportion } \\
(\%)\end{array}$} \\
\hline & Active & Inactive & Total & & Active & Inactive & Total & \\
\hline 60 & 19.01 & 2.51 & 21.52 & 88.34 & 20.08 & 4.15 & 24.23 & 82.86 \\
\hline 65 & 14.81 & 2.51 & 17.32 & 85.51 & 15.79 & 4.16 & 19.95 & 79.17 \\
\hline 70 & 10.99 & 2.50 & 13.49 & 81.46 & 11.81 & 4.12 & 15.93 & 74.12 \\
\hline 75 & 7.66 & 2.47 & 10.13 & 75.59 & 8.26 & 4.03 & 12.29 & 67.21 \\
\hline 80 & 4.94 & 2.42 & 7.36 & 67.12 & 5.30 & 3.84 & 9.14 & 57.95 \\
\hline 85 & 2.88 & 2.33 & 5.21 & 55.31 & 3.04 & 3.53 & 6.57 & 46.24 \\
\hline 90 & 1.47 & 2.19 & 3.66 & 40.27 & 1.52 & 3.10 & 4.62 & 32.91 \\
\hline 95 & 0.64 & 1.99 & 2.63 & 24.35 & 0.66 & 2.60 & 3.26 & 20.29 \\
\hline 100 & 0.24 & 1.74 & 1.98 & 12.18 & 0.26 & 2.10 & 2.36 & 11.13 \\
\hline
\end{tabular}

Table 7. Age-specific healthy and unhealthy life expectancies of the elderly in the central region.

\begin{tabular}{|c|c|c|c|c|c|c|c|c|}
\hline \multirow{3}{*}{ Age } & \multicolumn{4}{|c|}{ Male } & \multicolumn{4}{|c|}{ Female } \\
\hline & \multicolumn{3}{|c|}{ Life expectancy (year) } & \multirow{2}{*}{ ALE proportion (\%) } & \multicolumn{3}{|c|}{ Life expectancy (year) } & \multirow{2}{*}{$\begin{array}{l}\text { ALE proportion } \\
(\%)\end{array}$} \\
\hline & Active & Inactive & Total & & Active & Inactive & Total & \\
\hline 60 & 17.02 & 2.60 & 19.62 & 86.75 & 17.66 & 5.16 & 22.82 & 77.37 \\
\hline 65 & 13.12 & 2.64 & 15.76 & 83.25 & 13.49 & 5.26 & 18.75 & 71.97 \\
\hline 70 & 9.63 & 2.69 & 12.32 & 78.17 & 9.62 & 5.32 & 14.94 & 64.38 \\
\hline 75 & 6.64 & 2.75 & 9.39 & 70.73 & 6.22 & 5.31 & 11.53 & 53.94 \\
\hline 80 & 4.23 & 2.82 & 7.05 & 59.99 & 3.49 & 5.13 & 8.62 & 40.47 \\
\hline 85 & 2.42 & 2.89 & 5.31 & 45.59 & 1.63 & 4.74 & 6.37 & 25.56 \\
\hline 90 & 1.21 & 2.92 & 4.13 & 29.24 & 0.63 & 4.14 & 4.77 & 13.11 \\
\hline 95 & 0.53 & 2.84 & 3.37 & 15.63 & 0.23 & 3.49 & 3.72 & 6.11 \\
\hline 100 & 0.23 & 2.65 & 2.88 & 8.09 & 0.10 & 2.90 & 3.00 & 3.20 \\
\hline
\end{tabular}

Table 8. Age-specific healthy and unhealthy life expectancies of the elderly in the western region.

\begin{tabular}{|c|c|c|c|c|c|c|c|c|}
\hline \multirow{3}{*}{ Age } & \multicolumn{4}{|c|}{ Male } & \multicolumn{4}{|c|}{ Female } \\
\hline & \multicolumn{3}{|c|}{ Life expectancy (year) } & \multirow{2}{*}{ ALE proportion (\%) } & \multicolumn{3}{|c|}{ Life expectancy (year) } & \multirow{2}{*}{$\begin{array}{c}\text { ALE proportion } \\
(\%)\end{array}$} \\
\hline & Active & Inactive & Total & & Active & Inactive & Total & \\
\hline 60 & 15.82 & 3.04 & 18.86 & 83.87 & 17.92 & 5.97 & 23.89 & 74.99 \\
\hline 65 & 12.29 & 3.11 & 15.40 & 79.80 & 13.80 & 5.94 & 19.74 & 69.91 \\
\hline 70 & 9.07 & 3.14 & 12.21 & 74.27 & 10.02 & 5.82 & 15.84 & 63.28 \\
\hline 75 & 6.27 & 3.11 & 9.38 & 66.87 & 6.75 & 5.56 & 12.31 & 54.85 \\
\hline 80 & 4.00 & 2.97 & 6.97 & 57.37 & 4.15 & 5.12 & 9.27 & 44.73 \\
\hline 85 & 2.32 & 2.71 & 5.03 & 46.08 & 2.31 & 4.52 & 6.83 & 33.77 \\
\hline 90 & 1.22 & 2.36 & 3.58 & 34.18 & 1.18 & 3.82 & 5.00 & 23.63 \\
\hline 95 & 0.61 & 1.97 & 2.57 & 23.62 & 0.59 & 3.13 & 3.72 & 15.95 \\
\hline 100 & 0.30 & 1.61 & 1.91 & 15.92 & 0.31 & 2.53 & 2.84 & 10.99 \\
\hline
\end{tabular}

expectancy was 21.52, 19.62 and 18.86 years, respectively, and the ALE was 19.01, 17.02 and 15.82 years, respectively. Moreover, the difference between the eastern and central regions was significantly greater than the difference between the central and western regions. The ILE for this same group was 2.51, 2.60 and 3.04 years 
in the eastern, central and western regions, respectively. These differences between regions were not significant, but the eastern region's ILE was less than that of the central region, which was less than that for the western region. The ALE proportions for the eastern, central, and western regions were $88.34 \%, 86.75 \%$ and $83.87 \%$, respectively. The regional differences among female elderly were similar to those for male elderly.

Figure 3(a) shows the male elderly life expectancies by region; Figure 3(b) shows the male elderly ALEs by region; and Figure 3(c) shows the male elderly ILEs by region. Figure 4 displays female elderly life expectancy, ALE and ILE.

The following conclusions can be drawn based on Figure 3 and Figure 4. 1) There were obvious differences in life expectancy and ALE among male elderly in the eastern, central, and western regions in the lower age

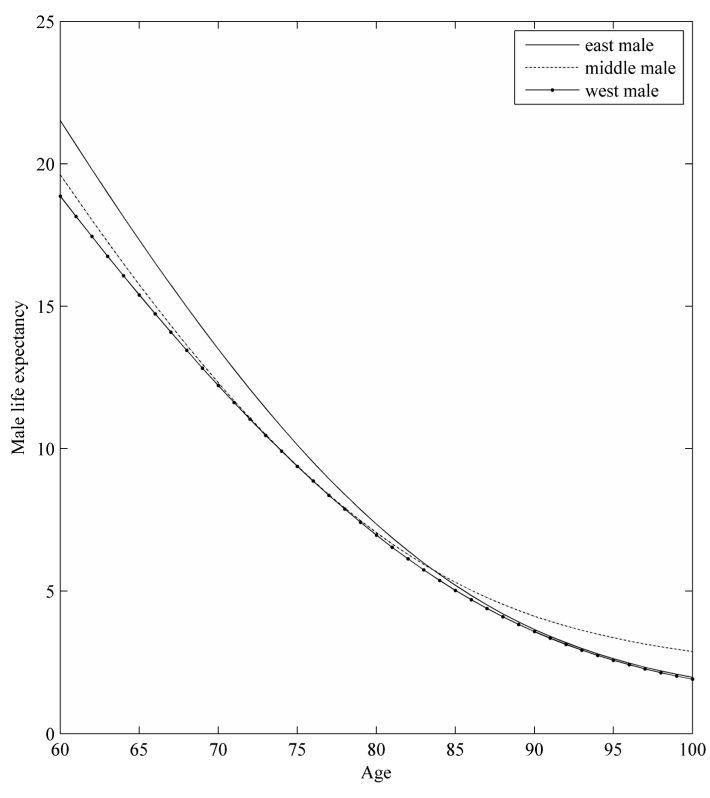

(a)

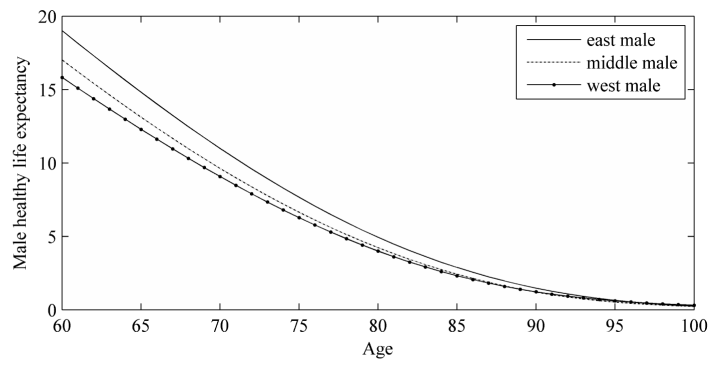

(b)

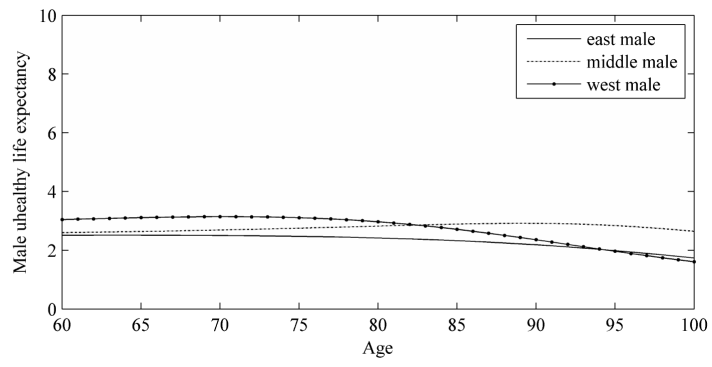

(c)

Figure 3. Life expectancy (a), ALE (b) and ILE (c) of male elderly by region.

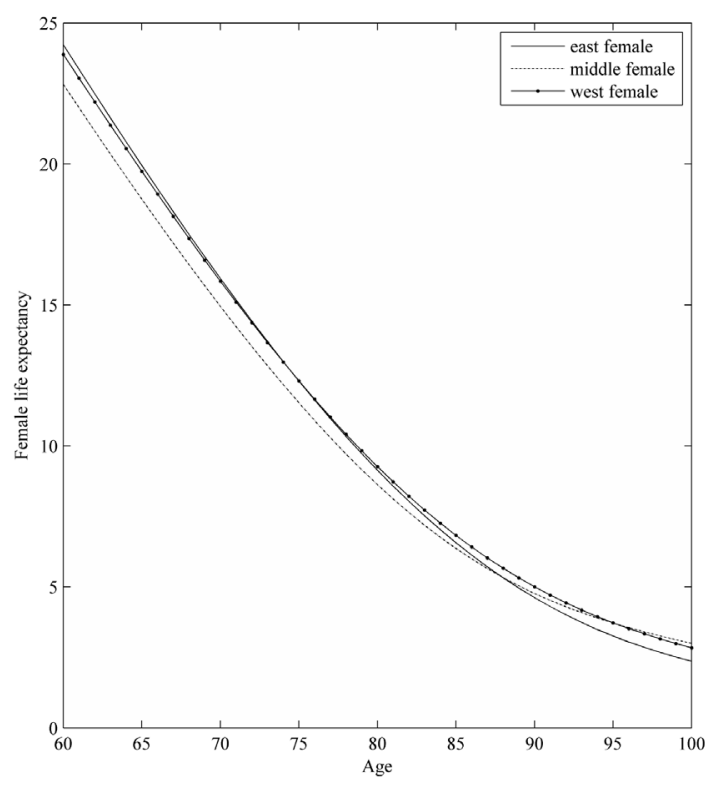

(a)

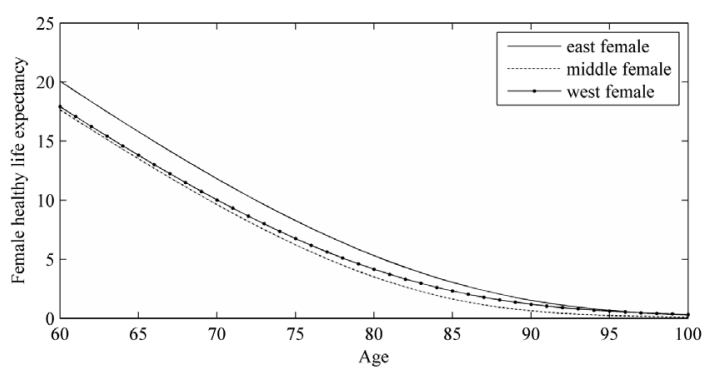

(b)

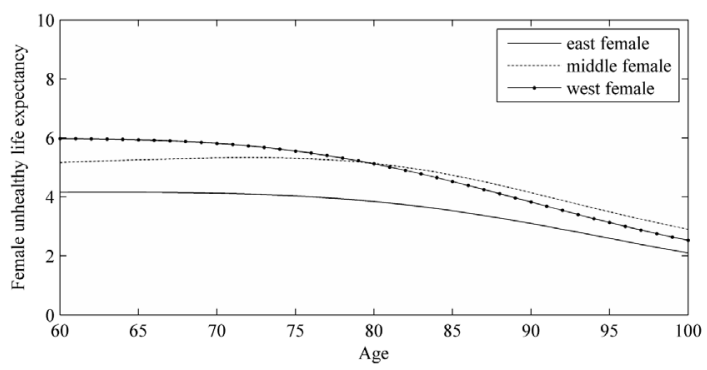

(c)

Figure 4. Life expectancy (a), ALE (b) and ILE (c) of female elderly by region. 
group. In particular, the values in the eastern region were higher than those for the central region, the central region's values were higher than those in the west, and the gap between regions decreased as age increased. In addition, there were no significant life expectancy or ALE differences among female elderly from the eastern, central, and western regions in the lower age group. Specifically, these values were slightly higher in the east than in the central and western regions, although the central region's values were lower than those for the west, and the gap between regions decreased as age increased. 2) ILEs among male elderly in the eastern, central, and western regions differed. In the lower age group, ILEs in the eastern region were shorter than those in the center of the country, and the central region's ILEs were shorter than those in the west. In the higher age group, however, there was a significant decrease in ILE among the western male elderly that eventually became shorter than the ILEs for the eastern and central elderly. The central male elderly's ILEs remained stable, and there was even a slight increase in the higher age group. The female elderly's ILEs were similar to those for males in the lower age group. Specifically, the values in the east were lower than those for the central region, and the central region's ILEs were shorter than those in the west, whereas in the higher age group, ILEs in the west were shorter than in the central region. 3) For the ALE proportions, those for the eastern region's males remained relatively high, and those for the central region's females were somewhat low.

The above analysis revealed differences in life expectancy and health conditions among male elderly in the eastern, central, and western regions of China, whereas there were no evident differences among females by region. The reasons for these findings may be as follows. First, we did not distinguish between urban and rural areas within regions; because these differences were significant, they could have led to differences in indicators that offset one another when we analyzed the mean values. Second, the regional division was not accurate in that it only reflected different regional economic development levels. The economic differences between the central and western regions are not significant compared with the eastern region, which could be one of the reasons that the life expectancies and health conditions of the elderly in the central and western regions did not differ significantly, especially among female elderly.

\section{Discussion}

In this paper, the health condition was divided into three states: Active, Inactive and Death, and the states were defined by ADLS: eating, dressing, bathing, using the toilet, indoor activities, and continence. In the questionnaires in 2006 and 2010, there are more indicators of the health condition, such as shopping, cooking, washing clothes, lifting weights about $10 \mathrm{~kg}$, managing finance, using the telephone, walking a long distance, walking up and down stairs, taking a bus. We can combine the ADLS and additional indicators together, and then the inactive state could be divided into two states, including partial self-care and complete lack of self-care, which would enable calculation of life expectancy in four states: healthy, partial self-care, complete lack of self-care and death. When we get the life expectancy of complete lack of self-care, we can evaluate the pension costs, which is so important to the elderly-care policy.

In addition, further research could focus on developing new models for analyzing ALE trends over time. In this paper, we used the IMaCh method to get the life expectancy in three states. When the health condition was divided into four states, we cannot use the same method to obtain the corresponding life expectancy, because the IMaCh needs more available data to get the final results. Since the data is limited, if we want to get the life expectancy in four states, we have to develop new method to resolve this problem.

\section{Conclusions}

We used tracking data from 2006 and 2010, obtained from surveys administered to Chinese elderly, to analyze their life expectancies and health conditions. Our analysis followed three dimensions, including gender, census register and region, and we presented the trends in life expectancy, ALE, ILE and ALE proportions along these three dimensions. Our main conclusions are as follows. 1) In general, life expectancy and ALE were significant lower for Chinese elderly males than for Chinese elderly females of the same age. In addition, both of these indicators decreased as age increased, and the gap in indicators between males and females decreased as well. 2) ILEs among male and female elderly remained approximately unchanged in the lower age group, and the ILE for females was longer than that for males, whereas a different tendency was observed in the higher age group. 3) Life expectancies and ALEs for female elderly were longer than those for male elderly, although the ALE proportions for males were greater than those for females, indicating that the male elderly's quality of life was 
better than that of females. 4) Life expectancy, ALE and ILE differed significantly between the urban and rural elderly groups; on all four indicators, the values for the urban elderly exceeded those of the rural elderly except the ILE of female. 5) By comparing the life expectancies and health conditions of the urban and rural elderly groups, we found that the increased life expectancy among the Chinese elderly in the lower age group was mainly attributed to the increased ALE, whereas in the higher age group, it was mainly related to the increased ILE. 6) The life expectancies and health conditions of the elderly in China did not differ significantly among the country's eastern, central, and western regions.

\section{References}

[1] Du, P. and Li, Q. (2006) Disability-Free Life Expectancy of Chinese Elderly and Its Change between 1994 and 2004. Population Research (Chinese), 30, 9-17.

[2] Carins, A.J., Blake, D.P. and Dowd, K. (2008) Modeling and Management of Mortality Risk: A Review. Scandinavian Actuarial Journal, 2008, 79-113. http://dx.doi.org/10.1080/03461230802173608

[3] MacMinn, R., Brockett, P. and Blake, D. (2006) Longevity Risk and Capital Markets. Journal of Risk and Insurance, 73, 551-557. http://dx.doi.org/10.1111/j.1539-6975.2006.00188.x

[4] Majer, I.M., Stevens, R., Nusselder, W.J., Mackenbach, J.P. and van Baal, P.H.M. (2013) Modeling and Forecasting Health Expectancy: Theoretical Framework and Application. Demography, 50, 673-697. http://dx.doi.org/10.1007/s13524-012-0156-2

[5] Katz, S., Ford, A.B., Moskowitz, R.W., Jackson, B.A. and Jaffee, M.W. (1963) Studies of Illness in the Aged: The Index of ADL, a Standardized Measure of Biological and Psychosocial Function. Journal of the American Medical Association, 185, 914-919. http://dx.doi.org/10.1001/jama.1963.03060120024016

[6] Pope, A.M. and Tarlov, A.R. (1991) Disability in America: Toward a National Agenda for Prevention. National Academy Press, Washington DC.

[7] World Health Organization (WHO) (2002) Towards a Common Language for Functioning, Disability and Health: ICF. WHO, Geneva. http://www.who.int/classifications/icf/training/icfbeginnersguide.pdf

[8] Verbrugge, L.M. and Jette, A.M. (1994) The Disablement Process. Social Science and Medicine, 38, 1-14. http://dx.doi.org/10.1016/0277-9536(94)90294-1

[9] Robine, J.M. and Jagger, C. (2003) Creating a Coherent Set of Indicators to Monitor Health across Europe: The Euro-REVES 2 Project. The European Journal of Public Health, 13, 6-14. http://dx.doi.org/10.1093/eurpub/13.suppl 3.6

[10] Robine, J.M., Jagger, C., Mther, C.D., Crimmins, E.M. and Suzman, R.M. (2003) Determining Health Expectancies. John Wiley \& Sons Ltd., Chichester.

[11] Gu, D.N. and Zeng, Y. (2006) Changes of Disability in Activities of Daily Living among Chinese Elderly from 1992 to 2002. Population \& Economics, 4, 9-14.

[12] Zeng, Y., Gu, D. and Land, K.C. (2004) A New Method for Correcting Underestimation of Disabled Life Expectancy and Application to Chinese Oldest-Old. Demography, 41, 335-361. http://dx.doi.org/10.1353/dem.2004.0018

[13] Zeng, Y., Gu, D. and Land, K.C. (2005) Correcting Underestimation of Disabled Life Expectancy: Methodology and Application. In: Zeng, Y., Crimmins, E., Carrire, Y. and Robine, J.-M., Eds., Longer Life and Healthy Aging, Springer, The Netherlands, 49-70.

[14] Gao, X.Y. and Kang, X.P. (2010) Analyzing the Health Life Expectancy of the Chinese Oldest-Old by Using the Multistate Life Table Method. Chinese Journal of Health Statistics, 127, 455-458.

[15] Zeng, Y., Gu, D. and Land, K.C. (2007) The Extension of the Method for Estimating the Health Life Expectancy and Its Application to the Chinese Oldest-Old. Chinese Journal of Population Science, 6, 2-14.

[16] Laditka, S.B. and Wolf, D.A. (1998) New Methods for Analyzing Active Life Expectancy. Journal of Aging and Health, 10, 214-241. http://dx.doi.org/10.1177/089826439801000206

[17] Sullivan, D.F. (1971) A Single Index of Mortality and Morbidity. HSMHA Health Reports, 86, 347-354. http://dx.doi.org/10.2307/4594169

[18] Crimmins, E.M. and Saito, Y. (2001) Trends in Healthy Life Expectancy in the United States, 1970-1990: Gender, Racial and Educational Differences. Social Science and Medicine, 52, 1629-1641. http://dx.doi.org/10.1016/S0277-9536(00)00273-2

[19] Crimmins, E.M., Saito, Y. and Ingegneri, D. (1997) Trends in Disability-Free Life Expectancy in the United States 1970-1990. Population and Development Review, 23, 555-572. http://dx.doi.org/10.2307/2137572

[20] Jiang, C., Gu, D.N., Liu, Y.Z. and Zeng, Y. (2009) Cost Analysis of Homecare and Nursing Home Services of the Chinese Elderly Based on Multistate Life Table. Population Research, 33, 81-88. 